\title{
La comprensión y recuerdo de textos expositivos a través del análisis de algunas variables del texto y lector
}

\author{
JOSÉ A. LEÓN \\ Universidad Autónoma de Madrid

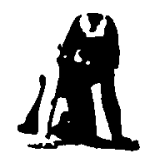 \\ Resumen
}

En el presente estudio se analizaron los posibles efectos de una variable del texto, su complejidad presentada en su forma léxica y semántica, podria ejercer en la comprensión y recuerdo del texto leido en grupos de sujetos con diferente nivel de conocimientos previos y destreza lectora. Los sujetos que participaron en el estudio fueron 48 expertos* (postlicenciados) y 96 "novicios* (alumnos de 1. de BUP), éstos últimos con dos niveles de comprensión, buenos y malos ( $48 B C$ y $48 M C$, respectivamente). Todos los sujetos leyeron dos textos con diferente nivel de dificultad comprensiva y realizaron dos tareas de recuerdo libre: inmediato y demorado (siete dias). Los resultados confirmaron que esta variable del texto fue clave para la comprensión final, ya que de ella dependía el comportamiento de los distintos grupos de lectores. Asi, aunque en general los resultados indicaron que los lectores aexpertos. obtuvieron las mejores puntuaciones, especialmente en la macroestructura del pasaje, tales diferencias aumentaban o disminuian en función de dicha variable textual.

Palabras clave: Comprensión de textos. Complejidad del texto. Expertos-novatos. Habilidades lectoras. Textos expositivos.

\section{Comprehension and recall of expository texts through the analisis of some text and reader variables}

\section{Abstract}

In the present study, we investigated the effects of the lexical and semantic complexity on the text comprehension and recall in different reader groups who differenciated in their comprehension and previous knowledge level about the topics of the text. Subjects were 48 *experts* (post-graduates students) and 96 anovices* (junior bigh school students), 48 good readers and 48 poor readers. All of the subjects read two expository texts at different levels of difficulty. Two recall tests were made, inmediate free recall and delayed free recall (seven days). The results demonstrated that this feature texts, complexity of the text, were an essential factor in the comprebension, since it decide the conduct of the different reader groups. So, althought the results in general demonstrated that the expert readers obtain the best scores, especially in the macrostructure, these results depend on the comprehension difficulty of the text.

Key words: Text comprebension. Complexity of the text. Skill reader. Expert-novice. Expository text.

Dirección del autor: Universidad Autónoma de Madrid. Facultad de Psicología. Departamento de Psicología Básica, Social y Metodología. Campus de Cantoblanco. 28049 Madrid. 1991.

Original recibido: noviembre 1990. Revisión recibida: julio 1991. Aceptado: septiembre 


\section{INTRODUCCION}

Numerosos trabajos teóricos y aplicados apoyan la idea de que la comprensión y el recuerdo que obtenemos de la información de un texto es el resultado que depende tanto de las características y estructura del propio pasaje, como del papel activo que desempeña el lector en su procesamiento (Justa y Carpenter, 1987; Oakhill y Garnham, 1988). Así, una carencia en alguno de estos factores puede repercutir de manera importante en una correcta comprensión y asimilación del contenido del texto.

Una fuente de los problemas o deficiencias en la comprensión puede tener su origen en el texto. Una de ellas hace referencia a que los textos estén pobremente estructurados. Kintsch y Yarbrough (1982), por ejemplo, pusieron de manifiesto que cuando el texto posee una estructura anómala, ello repercute negativamente en su comprensión. No en vano, la intervención educativa ha dirigido sus esfuerzos hacia la mejora de los textos dirigiendo buena parte de estos trabajos hacia textos expositivos. Una razón de esta elección es que estos textos, al transmitir una gran cantidad de información nueva para el lector, resultan difíciles de asimilar.

Desde esta misma perspectiva, el lector aporta dos aspectos claves en el procesamiento del discurso, su conocimiento previo y sus habilidades o estrategias necesarias para comprender, retener y aplicar la información extraída del material escrito. Autores como Spillich, Vesonder, Chiesi y Vos (1979) ya demostraron en diferentes trabajos el enorme impacto que los conocimientos previos sobre la temática del texto tiene sobre su comprensión. Pero no sólo sobre el contenido del texto. También el conocimiento sobre la estructura interna del pasaje expositivo, facilita de manera importante su comprensión y posterior recuerdo (Meyer y cols., 1980; Taylor y Samuels, 1983; Garner y cols., 1986).

También la investigación se ha centrado en la evaluación de la capacidad estratégica de los alumnos cuando se enfrentan ante un material escrito. En ellas se han descubierto importantes diferencias en el uso de estrategias de procesamiento entre lectores "expertos" y "novicios» (Meyer, 1975, 1984; Meyer, Brandt y Bluth, 1980; Vipond, 1980; Walker y Meyer, 1980; Meyer y Rice, 1982; Loman y Mayer, 1983; Rahman y Bisanz, 1986; León y Carretero, 1990). Los buenos lectores tienden a utilizar una «estrategia estructural» (structural estrategy) consistente. Identifican el contenido superordinario y la forma jerárquica del texto en su memoria a la vez que suelen suprimir la información complementaria y redundante en la tarea de recuerdo. Los lectores menos com- petentes, por el contrario, tienden a utilizar un "estrategia de lista». Otorgan la misma importancia a todo el contenido, mostrándose incapaces de distinguir entre diferentes niveles del texto.

Sin embargo, a pesar de estos descubrimientos, no son muchos los estudios que traten ambos aspectos simultáneamente y se pueda con ello, precisar y analizar posibles situaciones que conducen a una pobre comprensión de lo leído. Debería profundizarse en el estudio de algunas de las características del lector, tales como el nivel de comprensión y conocimientos previos (estudios sobre expertos-novicios), así como en algunas de las características de los textos, como es la dificultad comprensiva de los mismos.

\section{Planteamiento e hipótesis}

El propósito fundamental de esta investigación fue la de analizar experimentalmente como una de las características del texto (complejidad) afecta al recuerdo de sujetos con distinto nivel de competencia lectora y nivel de conocimientos previos. Sobre esta base, nos interesamos en investigar sobre los siguientes aspectos:

Un primer objetivo fue el establecimiento previo de tres niveles de competencia lectora siguiendo la línea de trabajos expertos-novatos: sujetos «novicios» pertenecientes al mismo nivel académico y diferente nivel de comprensión lectora (Sujetos BC y MC) y sujetos «expertos» tanto en habilidades lectoras como en conocimientos previos (Licenciados en Geografía e Historia). Nos planteamos cómo estos tres distintos niveles se comportarian ante diferentes tareas lectoras.

Un segundo objetivo se centró en el tipo de texto presentado. Deseabamos comprobar experimentalmente cómo la dificultad comprensiva de los textos seleccionados, expresada ésta en una mayor complejidad léxica y semántica, repercutiria en el comportamiento lector de los diferentes grupos de sujetos. Para ello, se seleccionaron dos textos expositivos que diferían en su contenido y que exigian al lector poseer un determinado nivel de conocimientos previos para poder obtener una comprensión correcta. El primero de ellos, «Superpetroleros», se simplificó léxica y semánticamente para que, junto a su temática familiar, resultase al lector un texto sencillo y cotidiano. Este pasaje sólo requeria para su comprensión un nivel general de conocimientos. Por el contrario, el segundo texto titulado «Los Orígenes del Descubrimiento de América», de mayor dificultad semántica y léxica, reflejaba una terminología más técnica, más adecuada al conocimiento del experto. Por ello, es esperable que los expertos 
obtuviesen diferencias aún mayores en el recuerdo de este segundo texto.

Por último, un tercer objetivo fue el de estudiar el efecto que el tipo de tarea de recuerdo producía en las respuestas de los sujetos. Utilizamos dos tareas diferentes: Tarea de recuerdo libre inmediato y tarea de recuerdo libre demorado ( 7 días). Estas tareas, muy utilizadas en trabajos precedentes, pretenden medir la información recordada en dos momentos diferentes, por considerar que intervienen distintos tipos de memoria y procesos. El recuerdo libre inmediato implicaría un proceso de codificación de la información y la posible intervención de la memoria a corto plazo, debido a la prontitud con la que se ejecuta la tarea. Por el contrario, en la tarea de recuerdo libre demorado (siete días más tarde), la información ya ha sido transferida a la memoria a largo plazo y el proceso interviniente sería en este caso la recuperación. Este último tipo de recuerdo expresaría qué información del texto ha sido capaz de asimilar el lector.

\section{Método}

\subsection{Sujetos}

Participaron en este estudio un total de 144 sujetos distribuidos de la siguiente forma: 48 lectores «expertos», licenciados en Geografía e Historia de la Universidad Autónoma de Madrid y 96 lectores «novatos» con diferentes niveles de comprensión lectora, 48 buenos lectores (BC) y 48 malos lectores (MC), todos ellos alumnos de $10^{\circ}$ de BUP y pertenecientes al Instituto de Bachillerato «Rey Pastor» de Madrid. Estos últimos niveles de competencia lectora se obtuvieron de una selección de 209 alumnos que fueron sometidos a tres tareas previas: a) una Prueba de comprensión lectora (Lázaro, 1982); b) una valoración del rendimiento académico de cada alumno por los profesores de Matemáticas, Naturales y Lengua y especialmente de Historia; y c) una tarea de resumen. A través de esta tarea se evaluaba la presencia o ausencia de las ideas importantes del texto, así como su coherencia global.

\subsection{Materiales}

Para este estudio se seleccionaron dos textos expositivos con un contenido distinto y bien diferenciado: "Los superpetroleros" y "Los orígenes del descubrimiento de América». La estructura de ambos textos fue elaborada de acuerdo al método de análisis de Meyer (1975, 1985), correspondiendo el primero a una adaptación española sobre la ya utilizada por la autora en investigaciones anteriores (Meyer y Rice, 1982). El segundo pasaje fue adaptado para este fin sobre un texto original de Céspedes del Castillo (1983). Los textos diferían en la relación retórica dominante, siendo en el caso de «los superpetroleros" de respuesta: problema-solución y de covarianza: antecedente-consecuente en el texto sobre el descubrimiento. Otra diferencia entre ambos fue el nivel léxico y semántico utilizado, siendo la del segundo más complejo y especializado.

Otras modificaciones que se realizaron en el material escrito, se mantuvieron constantes. Tal es el caso del contenido, similar en extensión y número de unidades ideacionales que los conforman. La extensión del texto «Superpetroleros» fue de 452 palabras con un total de 204 unidades ideacionales. El segundo contaba con 436 palabras y un total de 199 unidades ideacionales. En ambos textos se establecieron 10 niveles jerárquicos, correspondiendo los cinco primeros al nivel macroproposicional y el resto al microproposicional.

\subsection{Procedimiento}

La evaluación consistió de dos sesiones. En la primera cada alumno recibía junto al texto que debía leer un folio en blanco en el que apuntaba sus datos y el código del texto. Los sujetos dispusieron de un tiempo máximo de 12 minutos de lectura. Se insistió en que la lectura del texto debería realizarse con atención puesto que después habría que realizar alguna tarea sobre el mismo. En ningún momento se explicitó cuál era la tarea que deberían realizar ni el tiempo que disponían para leerlo. Una vez concluido el tiempo, que recogieron los textos presentados y se les pidió que tratasen de recordar todo lo que pudiesen del texto que se acababa de leer. El tiempo máximo que se les permitió en esta fase fue de 15 minutos. Todos los tiempos empleados fueron consignados como válidos en un estudio piloto.

En una segunda sesión, exactamente una semana después y sin previo aviso, se les invitó de nuevos a que tratasen de recordar todo lo que fueran capaces de los textos leídos en la semana anterior. El tiempo máximo fue idéntico al de la segunda sesión, 15 minutos.

\subsection{Puntuación}

Para medir el recuerdo de cada lector, se siguió el análisis de la estructura del texto elaborada por Meyer (1985). Los datos finales de cada sujeto se agrupaban en torno a cuatro índices cuantitativos y uno cualitativo. Fueron los siguientes:

\section{Indices Cuantitativos:}

- Número de ideas (puntuaciones A) correspondientes a los niveles más altos (1-5). 
Representan la macroestructura del texto. El texto de los superpetroleros contiene 47 palabras; el segundo texto, 27.

- Número de ideas de detalle (puntuaciones B) correspondientes a los niveles más bajos (6-10). Mientras el texto de Meyer suma 54, el texto sobre América incluye 67.

- Número de relaciones retóricas básicas (puntuaciones R). Corresponden a aquellas relaciones que conforman la estructura principal y el nivel macroestructural del pasaje y que fueron las siguientes: respuesta: problema-solución, causación: antecedente-consecuente, comparación, descripción y colección. Se consideran básicas las situadas en los cinco primeros niveles de la estructura de contenido de cada texto. Para el primer texto comprenden un total de 22 y 14 para el segundo.

- Número total de ideas recordadas (puntuaciones G). Es la suma de las tres puntuaciones anteriores. El texto de los superpetroleros contiene un total de 123 unidades por 108 el texto sobre América.

\section{Indice Cualitativo (0)}

A través de esta puntuación se trataba de evaluar el grado de concordancia con el que los sujetos orientaban su recuerdo siguiendo la estructura interna del texto. En base a este índice, establecimos tres niveles: Cuando el lector sigue en la exposición de su recuerdo la estructura retórica de alto nivel del texto y los principales elementos inmediatamente por debajo (problema-solución, colección de soluciones...) (V puntos); cuando el sujeto, aunque sigue la organización retórica del texto, ésta no se expresa de manera explícita, apareciendo algunos de sus elementos básicos (III puntos). Finalmente, cuando en el recuerdo no se expresa ni de forma central ni incidental los elementos retóricos fundamentales (I punto). Tal es el caso de recuerdo poco coherente, donde aparecen algunos hechos aislados que tratan de hilar algún detalle.

Los protocolos fueron corregidos por dos evaluadores. El criterio utilizado para evaluar el acuerdo interjueces en las puntuaciones cuantitativas fue el coeficiente Kappa de Cohen ( $k$ ). Este coeficiente fluctuó entre $k=0,75$ y $\mathrm{k}=0,81$, correspondiendo el más bajo a los sujetos expertos, más difíciles de jugar por la riqueza del recuerdo y el gran número de inferencias realizadas. En cualquier caso, todos los valores consignados se situaron en el nivel más alto de la escala. El grado de acuerdo interjueces en la puntuación cualitativa fue de un $90 \%$, llegándose normalmente a un consenso en las diferencias encontradas.

\subsection{Diseño}

El análisis estadístico consta de dos diseños factoriales mixtos. El primer análisis consta de un diseño factorial muxto $3 \times 2 \times 2$, con sólo un factor intersujeto, grupo lector, y dos factores intrasujetos, texto y tarea de recuerdo, conformando 4 ANOVAs, uno para cada una de las puntuaciones medidas. El segundo análisis consta de un diseño factorial mixto de $3 \times 2$, también con un factor intersujeto, el grupo de lectores, y uno intrasujeto, el tipo de tarea de recuerdo. En un segundo apartado se han tratado de puntuaciones (0). En este caso se trata de puntuaciones que requieren un análisis cualitativo.

\section{Resultados}

\section{A) PUNTUACIONES A, B, R y G}

\subsection{Grupo de lectores}

Como era esperable, se han detectado diferencias significativas entre los distintos grupos de lectores $(p<0,001)$, siendo los sujetos más capaces los que mejores resultados han obtenido. Ahora bien, aunque parece evidente que la mejora en el recuerdo del texto está en función de la habilidad del lector, los resultados fluctúan dependiendo de la presencia de la dificultad del texto.

\subsection{Complejidad del texto}

La complejidad del texto aparece como un factor determinante en la comprensión y recuerdo del material escrito presentado. En efecto, si analizamos los datos obtenidos por los distintos grupos en cada uno de los textos por separado (váase Tabla 1), observamos que el comportamiento de los diferentes grupos de lectores varía considerablemente al texto de Meyer (Superpetroleros), son los alumnos hábiles de Bachillerato (BC) los que mejoran sus puntuaciones llegando a igualarse con la de los sujetos adultos respecto a la información relevante, y superan con facilidad al grupo de expertos en la información complementaria, medida en puntuaciones $B(p<0,01)$. Por el contrario, si nos referimos al segundo de los pasajes, "Los Orígenes del Descubrimiento», son los expertos los que aumentan sus diferencias respecto a la información relevante, como se muestra en las puntuaciones A y $R$, siendo significativa esta última $(p<0,05)$.

Los sujetos menos capaces (MC), tal y como se esperaba, se muestran claramente inferiores respecto a los dos grupos más hábiles en todas las puntuaciones y textos analizados $(p<0,001)$. Retienen comparativamente me- 
TABLA I

Contraste de medias correspondiente al cómputo de cada uno de los textos en los distintos indices de medida utilizados. El nivel de significación expresado en $\mathrm{p}$ ba sido hallado con el procedimiento de Scheffé. Los espacios señalados con guión indican ausencia de significación

Texto 1 (SUPERPETROLEROS)

\begin{tabular}{lcccc}
\hline & $\mathbf{A}$ & $\mathbf{B}$ & $\mathbf{R}$ & $\mathbf{G}$ \\
\hline EXP $<$ BC & $\overline{-}$ & 0.0033 & $\overline{-}$ & $\overline{-}$ \\
EXP $>$ MC & 0.0001 & $\overline{-}$ & 0.0001 & 0.0010 \\
BC $>$ MC & 0.0001 & 0.0005 & 0.0001 & 0.0001 \\
\hline
\end{tabular}

Texto 2 (ORIGENES DEL DESCUBRIMIENTO)

\begin{tabular}{lcccc}
\hline & A & B & R & G \\
\hline EXP > BC & 0.0881 & - & 0.0347 & - \\
EXP > MC & 0.0001 & 0.0001 & 0.0001 & 0.0001 \\
BC > MC & 0.0001 & 0.0001 & 0.0001 & 0.0001 \\
\hline
\end{tabular}

nos información, sea cual sea el nivel de ésta. $\mathrm{Su}$ tendencia en el recuerdo parece inclinarse más bien a retener algunas ideas de detalle, como muestra la única ausencia de significación respecto a los sujetos expertos en las puntuaciones $\mathrm{B}$ en el texto «Superpetrolerosn.

Esta divergencia en el comportamiento de los distintos grupos, ha producido, también desde esta perspectiva, interacciones significativas entre estos dos factores, grupo lector y texto, en todas las puntuaciones analizadas, siendo significativas en $R(p<0,05)$ y altamente significativas en $A, B$ y $G(p<0,001)$. Por último, se produce una interacción significativa entre el texto y las dos tareas del recuerdo en las puntuaciones $R(p<0,05)$. Tomando el cómputo global del todos los sujetos, las puntuaciones $R$ obtenidas en el texto sobre los superpetroleros fueron ligeramente superiores a las obtenidas en el pasaje sobre el descubrimiento en la tarea de recuerdo libre inmediato. Este hecho se invierte cuando analizamos la tarea de recuerdo demorado. La razón de que la interacción haya resultado significativa, parece residir en el comportamiento que los sujetos más hábiles mostraron en estas puntuaciones, especialmente en el segundo pasaje. Es en estas puntuaciones donde los expertos mejoran ostensiblemente su recuerdo respecto a las mismas puntuaciones obtenidas en el texto de Meyer. Los datos reflejan el papel relevante que el conocimiento previo ejerce en la retención de la información retórica del material escrito. El resultado final de los distintos grupos está en función de la adecuación de sus conocimientos a las características del texto, ya sean éstas el nivel de complejidad léxica y semántica, o la peculiaridad del contenido, siendo éste más general, familiar o específico y complejo.

\subsection{Tarea de recuerdo: Libre inmediato/libre demorado}

Tal como era de esperar, la tarea de recuerdo libre inmediato es netamente superior a la tarea de recuerdo libre demorado (siete días) en todos los tratamientos experimentales y en todas las puntuaciones analizadas $(p<0,001)$. Sin embargo, se han detectado importantes variaciones en el patrón de recuerdo característico de los sujetos expertos respecto a los lectores de bachillerato en las dos tareas de recuerdo realizadas, siendo el de los primeros más estables y resistente con el paso del tiempo.

Esta estabilidad es aún mayor cuando se trata de evocar la información relativa a la macroestructura y a las principales relaciones retóricas una semana después de la lectura de los pasajes. En efecto, como indican los diversos contrastes de medias realizadas (ver Tabla 2), de una cierta superioridad, aunque no significativa, del grupo BC en la tarea de recuerdo libre inmediato, se pasa a un aumento de las puntuaciones de los lectores expertos en los índices esenciales en la tarea demorada. El recuerdo de los expertos se muestra más resistente al paso del tiempo en la información relevante, existiendo diferencias significativas respecto al grupo $B C$ en las puntuaciones $R$ $(p<0,05)$ en el texto de Meyer y las puntuaciones $A(p<0,001)$ y $R(p<0,05)$ en el texto sobre el descubrimiento de América. Este mismo efecto se constata respecto a los sujetos 
TABLA II

Contraste de medias correspondiente a cada uno de los textos por separado en los distintos indices de medida utilizados $y$ en las dos tareas de recuerdo. El nivel de significación en $\mathrm{p}$ ba sido hallado siguiendo el procedimiento de Scheffé. Los espacios señalados con guión indican ausencia de significación

Texto 1 (SUPERPETROLEROS)

\begin{tabular}{|c|c|c|c|c|c|c|c|c|}
\hline & \multicolumn{4}{|c|}{ Rdo. inmediato } & \multicolumn{4}{|c|}{ Rdo. demorado } \\
\hline & $\mathbf{A}$ & B & $\mathbf{R}$ & G & $\mathbf{A}$ & B & $\mathbf{R}$ & G \\
\hline $\begin{array}{l}\operatorname{EXP}>\mathrm{BC} \\
\operatorname{EXP}<\mathrm{BC} \\
\operatorname{EXP}>\mathrm{MC} \\
\mathrm{BC}>\mathrm{MC}\end{array}$ & $\begin{array}{l}\bar{z} \\
0.001 \\
0.001\end{array}$ & $\begin{array}{l}\overline{0.001} \\
\overline{0.001}\end{array}$ & $\begin{array}{c}\overline{-} \\
0.001 \\
0.001\end{array}$ & $\begin{array}{l}\overline{0.001} \\
0.05 \\
0.001\end{array}$ & $\begin{array}{l}\bar{Z} \\
0.001 \\
0.001\end{array}$ & $\frac{\overline{0.05}}{0 . \overline{001}}$ & $\begin{array}{l}0.05 \\
0.001 \\
0.001\end{array}$ & $\begin{array}{l}\overline{-} \\
0.001 \\
0.001\end{array}$ \\
\hline
\end{tabular}

Texto 2 (ORIGENES DEL DESCUBRIMIENTO)

\begin{tabular}{|c|c|c|c|c|c|c|c|c|}
\hline & \multicolumn{4}{|c|}{ Rdo. inmediato } & \multicolumn{4}{|c|}{ Rdo. demorado } \\
\hline & $\mathbf{A}$ & B & $\mathbf{R}$ & $\mathbf{G}$ & $\mathbf{A}$ & B & $\mathbf{R}$ & G \\
\hline $\begin{array}{l}\operatorname{EXP}>\mathrm{BC} \\
\mathrm{EXP}>\mathrm{MC} \\
\mathrm{BC}>\mathrm{MC}\end{array}$ & $\begin{array}{l}\overline{-} \\
0.001 \\
0.001\end{array}$ & $\begin{array}{l}\overline{-} \\
0.001 \\
0.001\end{array}$ & $\begin{array}{l}\overline{0} \\
0.001 \\
0.001\end{array}$ & $\begin{array}{l}\overline{0} \\
0.001 \\
0.001\end{array}$ & $\begin{array}{l}0.001 \\
0.001 \\
0.001\end{array}$ & $\begin{array}{l}\overline{\bar{n}} \\
0.001 \\
0.001\end{array}$ & $\begin{array}{l}0.05 \\
0.001 \\
0.001\end{array}$ & $\begin{array}{l}\overline{-} \\
0.001 \\
0.001\end{array}$ \\
\hline
\end{tabular}

$\mathrm{MC}$, sólo que en este caso las diferencias en estas puntuaciones son aún mayores $(\mathrm{p}<0,001)$.

\section{B) PUNTUACIONES CUALITATIVAS (Puntuaciones 0)}

\subsection{Organización del recuerdo}

Con este indice pretendíamos analizar el grado de aproximación con el que los diversos grupos han organizado su recuerdo siguiendo la estructura del texto, la relación retórica de alto nivel, seguida de sus elementos principales.

\section{a) Organización del recuerdo en los diferentes grupos estudiados}

Son los adultos los que mejor planifican sus respuestas siguiendo la estructura del texto en todas las situaciones estudiadas. Tomando conjuntamente las puntuaciones V y III, se detectan importantes diferencias entre los grupos tratados. El grado de utilización de la estructura textual es muy alta en estos lectores y fluctúa, según las diferentes situaciones, entre un $62,5 \%(12,5 \%+50 \%)$ y un $100 \%$ $(66,6 \%+33,3 \%)$. Les siguen los sujetos $\mathrm{BC}$, cuyos porcentajes varían entre un $33,3 \%$ $(0 \%+33,3 \%)$ y un $91,7 \%$ $(12,5 \%+79,2 \%)$. Por último, los alumnos MC son los que obtienen los resultados más bajos, oscilando éstos entre un $0 \%$
$(0 \%+0 \%)$ y un $58,3 \%(0 \%+58,3 \%)$. Estos datos, estrechamente relacionados con los obtenidos anteriormente, indican que esta estrategia para identificar y utilizar la organización de alto nivel es una habilidad crucial que poseen los lectores adultos y que utilizan cuando tratan de comprender un material escrito.
b) El conocimiento previo y la complejidad del texto

La destreza del lector maduro no se refleja únicamente en esta habilidad para organizar sus respuestas siguiendo la estructura del texto. Otro hecho que se desprende de estos resultados es la relación entre el nivel de conocimientos previos del lector y su adecuación a la temática y especificidad del contenido del texto, tal y como se refleja en la calidad y consistencia del recuerdo. En efecto, cuando los lectores difieren sólo en la destreza lectora, esto es, cuando el pasaje presentado no requiere poseer un nivel de conocimientos específicos para ser comprendido, como es el texto sobre los superpetroleros, los resultados sobre la coherencia del recuerdo son más parejos entre los sujetos expertos y buenos lectores de bachillerato. Tomando conjuntamnete las puntuaciones $\mathrm{V}$ y III de cada grupo, los expertos alcanzan un porcentaje de un $75 \%(20,8+54,2)$ frente a un $75 \%(0+75)$ de los $\mathrm{BC}$ y de tan sólo un $28,8 \%(0+28,8)$ para los sujetos MC. Pero si 
introducimos un nuevo factor, un texto cuyo contenido requiera un conocimiento más específico para ser comprendido correctamente, como es el caso del texto sobre América, las diferencias entre los expertos y los sujetos de BUP se incrementan, especialmente en las puntuaciones obtenidas en la tarea con demora; $75 \%(25+50)$ de los lectores expertos frente a un $33,3 \%(0+33,3)$ y un $0 \%(0+0)$ de los grupos BC y MC, respectivamente. Estos datos parecen confirmar una vinculación muy estrecha entre el conocimiento previo del sujeto, el nivel de especificidad del contenido del texto y la calidad y consistencia del recuerdo obtenido en una tarea lectora.

\section{Discusión}

Los resultados obtenidos están en consonancia con lo esperado. Las variables introducidas en este estudio son determinantes en el comportamiento final de los lectores. Así, por ejemplo, las variables que introducimos del lector, nivel de comprensión lectora y nivel de conocimientos previos, determinan tres claros niveles de competencia. En efecto, de manera relativamente independiente a la variable textual introducida en este estudio, sólo los lectores expertos hacen uso de la estrategia estructural descrita por Meyer (1984). Estos sujetos son los que, en general, han obtenido los mejores resultados que podemos resumir en los siguientes puntos:

- Han mostrado una habilidad selectiva para identificar y retener las relaciones retóricas y la información relacionada con la macroestructura del texto. No atienden de la misma manera la información de detalle (puntuaciones B), en la que suelen puntuar más bajo que el resto de las puntuaciones analizadas. Esta habilidad selectiva mejora significativamente cuando se enfrentan a textos que se adecúan mejor a sus conocimientos específicos.

- Su patrón de recuerdo, como se demuestra en la tarea demorada, se comporta como el más estable de los grupos estudiados, especialmente en la información relevante, representada por las puntuaciones A y $R$.

- Han mostrado una extraordinaria habilidad para organizar su recuerdo de acuerdo al plan de organización que sigue el texto, independientemente de la variable textual aplicada. En este sentido, muestran implícitamente un conocimiento previo sobre la estructura del texto, a través del cual, ubican la nueva información dentro de sus huecos y valores ausentes, reteniendo fundamentalmente la información crucial del texto leído.

Los lectores adolescentes, en cambio, no parecen utilizar por sí mismos esta habilidad, sino que su rendimiento depende de la presencia de otros factores, como ha sido la dificultad comprensiva. Los alumnos más competentes (BC), por ejemplo, aunque poseen una importante capacidad retentiva que supera a veces a la de los lectores expertos en la tarea de recuerdo inmediato, no son selectivos respecto a la información relevante, como lo demuestran sus altas puntuaciones en $B$. Retienen de manera indiscriminada toda la información de la que son capaces. Así, si los sujetos expertos a la hora de recuperar la información del material escrito se preguntasen: ¿Qué información de la macroestructura del pasaje me resta por anadir?, los sujetos BC harían lo propio preguntándose, ¿qué información de todo el texto me falta por escribir?

Sin embargo, a diferencia del lector experto que parece tener automatizadas sus estrategias de comprensión, en el lector adolescente $\mathrm{BC}$, influye de manera importante en su rendimiento final la presencia de la variable textual tratada. Así, su rendimiento lector alcanza sus máximos cuando el texto presentado se adecúa a sus conocimientos generales, como es el caso del pasaje "superpetroleros", disminuyendo sus diferencias en el recuerdo respecto al conseguido por los lectores adultos en este mismo texto. Sólo en este caso han manifestado poseer cierta habilidad para organizar su recuerdo conforme al plan organizativo del texto. Sin embargo, el hecho de que estas mejoras sólo se produzcan en las situaciones más favorables junto a una pérdida importante de la información relevante cuando se desea recuperarla algún tiempo después, consideramos que estos lectores no tienen aún asumida dentro de sus habilidades lectoras «la estrategia estructural». No muestran, a diferencia del experto, algún tipo de conocimiento sobre la estructura del texto.

El grupo MC se ha comportado según lo esperado. Sus puntuaciones han sido, en general, muy bajas en todas las medidas realizadas, alcanzando en B sus puntuaciones más altas. No sólo no discriminan, como en el caso anterior, la información relevante, sino que optan por la más irrelevante. Al igual que sus compañeros de BUP, obtienen sus mejores puntuaciones cuando se enfrentan a la lectura de un pasaje más adecuado a sus conocimientos generales (superpetroleros). También su patrón de recuerdo es similar al de los BC. Se produce un decaimiento brusco en la tarea demorada en todas las puntuaciones analizadas. La diferencia estriba en la cantidad de información retenida, siendo ésta significativamente inferior a la del resto de los grupos en todas las medidas realizadas.

Los datos reflejan una enorme dificultad por parte de estos lectores en retener las principa- 
les relaciones retóricas del contenido del texto. Los sujetos MC claramente no utilizan la estructura del texto como forma de organizar el recuerdo. Tienden más bien a listar frases aisladas que ellos recuerdan del pasaje sin ningún tipo de interrelación o conexión entre ellas. Este comportamiento se identificaría con la estrategia «lista por defecto" (default strategy) propuesta por Meyer (1984) o la estrategia «Tema + Detalles». descrita por Scardamalia y Bereiter (1984).

\section{CONCLUSIONES}

Este trabajo confirma la existencia de una estrecha relación entre las características del texto (complejidad del texto) y del lector (Nivel de conocimiento sobre la temática del texto y nivel de destreza lectora), de tal manera que la presencia/ausencia de una de ellas repercute en. las otras.

Como consecuencia de lo anterior, también se detecta una relación entre la estructura organizativa del texto y el procesamiento de la información del pasaje en la memoria del sujeto. Esta relación entre las estructuras de los textos y los procesos de comprensión implica una capacidad estratégica diferente en los lectores.
Los lectores expertos inciden espontáneamente en tales estructuras y las aplican en la reconstrucción del significado en la memoria. Por el contrario, los lectores novicios o con menor capacidad desconocen o no aprovechan de esas propiedades presentes en el texto $y$, como consecuencia, su representación del significado carece de orden y coherencia. Esta habilidad para organizar el recuerdo siguiendo la estructura principal del texto, parece ser una característica exclusiva del lector maduro y, que de ser cierto, podría ser utilizada como un excelente predictor del recuerdo y del grado de comprensión que está obteniendo el lector (Meyer, 1987; Meyer y cols., 1989).

La complejidad léxica y sintáctica del texto y el nivel de conocimientos previos del lector se revelan como factores estrechamente relacionados. Tal es así, que pensamos que una correcta adecuación entre estos dos factores podría facilitar enormemente la activación de los procesos de comprensión y la obtención de una representación adecuada de lo leído, especialmente en los lectores más jóvenes. Por ello, consideramos que estos factores deberían ser tenidos en cuenta por el escritor o el docente, especialmente en aquellos casos en los que los textos forman parte de contenidos educativos, como es el caso de los libros de texto.

\section{Referencias}

Céspedes del Castillo, G. (1983). "América hispánica (1492-1898)". En M. Tuñón de Lara (ed.), Historia de España, tomo VI. Barcelona: Labor.

Garner, R.; AleXander, P.; Slater, W.; Hare, V. C.; SMith, T., y ReIS, R. (1986). *Children's Knowledge of structural properties of expository texts". Journal of Educational Psychology, 78, 411-416.

Just, M. A., y CARPENTER, P. A. (1987). The Psychology of Reading and Language Comprehension. Massachusetts: Allyn and Bacon.

KINTSCH, M., y YARBROUGH, J. C. (1982). «Role of thetorical structure in text comprehension». Journal of Educational Psychology, 75, 828-834.

LÁzARO, A. (1982). Prueba de comprensión lectora. Madrid: TEA.

LEÓN, J. A., y CARRETERO, M. (1990). *The text reader interaction in the comprehension and recall of expository texts. Workshop". Text processing from different perspectives. EARLI: Universidad de Amsterdam.

LOMAN, N. C., y MAYER, R. (1983). «Signaling Techniques that increase the understanbility of expository prosem. Journal of Educational Psychology, 75, 402-412.

MEYER, B. J. E. (1975). The organization of prose and its effects in memory. Amsterdam: North-Holland.

- (1984). "Text dimensions and cognitive processing». In H. Mandl, N. L. Stein, \& T. Trabasso (eds.), Learning and comprebension of text. Hillsdale, Nueva Jersey: Erlbaum.

- (1985). «Prose analysis: Purposes, procedures and problems». In B. K. Britton \& J. B. Black (eds.), Understanding Expository Text. Hillsdale, Nueva Jersey: Erlbaum.

- (1987). *Following the author's top-level organization: an important skill for reading comprehension». En R. J. Tierney, P. L. Anders y J. N. Mitchell (eds.), Understanding reader's understanding: Theory and Practice. Hillsdale, Nueva Jersey: Erlbaum.

MEYER, B. J. F.; BRANDT, D. M., y BLUTH, G. J. (1980). «Use of top-level structure in text: Key for reading comprehension of ninth-grade students». Reading Research Quarterly, 16, 72-102.

MEYER, B. J. F., y RICE, G. E. (1982). "The interaction of reader strategies and the organization of text". Text, 2, 155-192. 
Meyer, B. J. F.; Young, C. J., y BARTLETT, B. J. (1989). Memory Improved. Reading and Memory Enhancement Across the Life Span Through Strategic Text Structures. Nueva Jersey: Erlbaum.

OAKHILL, J., y GARNHAM, A. (1988). Becoming a Skilled Reader. Nueva York: Basil Blackwell.

RAHMAN, T., y BISANZ, G. L. (1986). «Reading ability and use of a story scheme in recalling and reconstructing information*. Journal of Educational Psychology, 78, 323-333.

SCARDAMALIA, M., y BeREITER, C. (1984). „Development of strategies in text processing*. En H. Mandl, N. L. Stein y T. Trabasso. Learning and comprehension of text. Hillsdale, Nueva Jersey: Erlbaum.

SPILICH, G. J.; VESONDER, G. T.; CHIESI, H. L., y VOSS, J. F. (1979). *Text processing of domain-related information for individuals with high - and low-domain knowledge. Journal of Verbal Learning and Verbal Behaviour, 18, 275-290.

TAYLOR, B. M., y SAMUELS, S. J. (1983). «Children's use of text structure in the recall of expository material. American Educational Research Journal, 20, 517-528.

VIPOND, D. (1980). *Micro-and macroprocesses in text comprehension*. Journal of Verbal Learning and Verbal Behavior, 19, 276-296.

WALKER, C H., y MEYER, B. J. F. (1980). «Integrating different types of information in text *. Journal of Verbal Learning and Verbal Bebavior, 19, 263-275.

\section{Extended summary}

Although in general it can be assumed that an interactive conception of reading comprehension exists (Just \& Carpenter, 1987; Oakhill \& Garnham, 1988), empirical support capable of simultaneosly analyzing some fundamental features of the reader and the text that improve the correct understanding and assimilation of the passage is not usual.

The fundamental proposal of this research was to experimentally analyze how a characteristic of the text (complexity of the text) affects the recall of the subjects with a different level of reading comprehension and previous knowledge. For this study, two expository texts with different contents were chosen: «The supertankers» and «The origins of this discovery of America». The structure of both texts was elaborated in agreement with the analysis method of Meyer $(1975,1985)$. The texts differed in lexical and semantic complexity, with the second text being much more complex and specialized.

A total of 144 subjects participated in this study. They were distributed in the following way: 48 «expert» readers, graduates in Geography and History and 96 «novice» readers with different levels of reading comprehension, 48 good readers and 48 poor readers. The «novice» readers were all in first year high school and attend school at a Public High School.

The results obtained are in agreement with what was expected. The existence of a close relationship between the characteristics of the text (complexity) and of the reader (level of Knowledge about the topic of the text and level of reading skill), in such a way that the presence/absence of one of them can have repercussions on the others. This relationship between the structures of the text and the processes of comprehension establish a different strategic capacity in the readers. The most capable or expert readers spontaneously take notice of such structures and they apply them to the reconstruction of the meaning in their memory. On the other hand, the less capable or novice readers do not know or do not take advantage of these properties present in the text and, as consequence their representation of the meaning lack order and coherence. 